\title{
Full-Face-Approach
}

\section{Der neue Trend in der Ästhetik}

— Der Trend bei ästhetischen Behandlungen zur Gesichtsrejuvenation mittlerweile hin zum Wiederaufbauen jugendlicher Konturen. Dazu ist ein umfassendes Behandlungskonzept unter Berücksichtigung mehrerer aufeinander abgestimmter Produkte erforderlich.

In der Ästhetik hat in den letzten Jahren ein Paradigmenwechsel stattgefunden. Denn Altersprozesse umfassen nicht nur die Haut, sondern auch tieferliegende Gewebeschichten bis zum Knochen, was therapeutisch berücksichtigt werden muss. Diese umfassende Behandlung ist nur durch eine Kombination verschiedener Maßnahmen möglich, wie z. B. der Behandlung mimischer Falten mit Botulinumtoxin A (z.B. Bocouture ${ }^{\circledR}$ ), der Wiederauffüllung von verlorengegangenem Volumen mit einem Volumenfiller und der abschließenden Korrektur von Restfältchen durch einen adäquaten Hyaluronsäurefiller.

Dr. Anthony V. Benedetto, Dermatologic SurgiCenter, Philadelphia (USA), betonte in seinem Vortrag die Wichtigkeit, vor der Behandlung zusammen mit den Patienten einen umfassenden Behandlungsplan inkl. Kosten festzulegen.

Der Full-Face-Approach ermöglicht Behandlungsergebnisse, die sonst nur durch operative Eingriffe erreicht werden können. Für eine rasche Patientenzufriedenheit werden in einer ersten Sitzung zunächst mimische Falten mit BTX-A behandelt. Im Abstand von zwei Wochen werden dann mit einem Volumenfiller bestehende Volumendefizite ausgeglichen sowie in einer weiteren Sitzung mit Dermalfillern auf Basis von $\mathrm{Hy}$ aluronsäure Restfältchen aufgefüllt.

Dr. Welf Prager, Dermatologikum Hamburg, demonstrierte in einer Livebehandlung das Vorgehen im Rahmen eines FullFace-Approaches. "Mit dem Gesichtsset, das die Firma Merz anbietet, wurde dem Wunsch der Ärzte entsprochen, Patienten eine Volumenbehandlung zu vertretbaren Preisen anbieten zu können", so Prager.

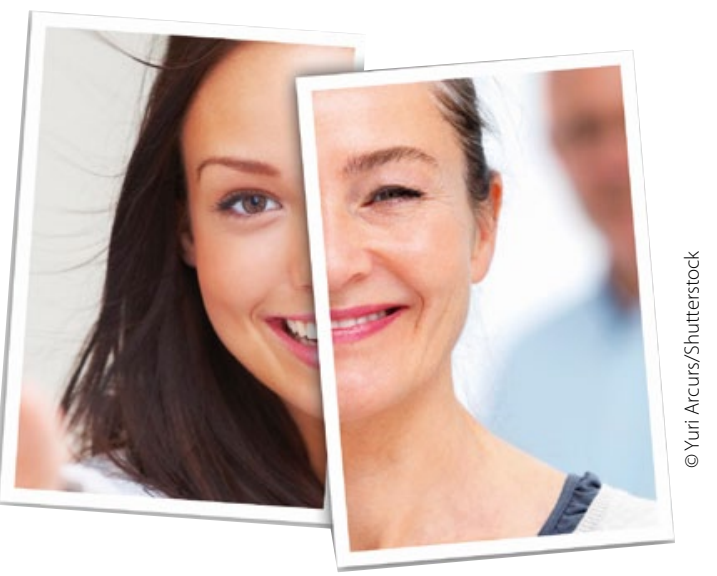

Die Rejuvenation des Gesichts muss im Alter auch unter der Haut befindliche Strukturen berücksichtigen.

Hier werden der Volumenfiller Radiesse ${ }^{\circledR}$ und der Hyaluronsäurefiller Belotero ${ }^{\oplus}$ als Set angeboten, wodurch ein attraktives Angebot für den Patienten möglich ist. Darüber hinaus gibt es weitere Angebote, die z. B. auch die Behandlung der Lippen oder der Tränenrinne mit Belotero ${ }^{\oplus}$ Basic einschließen.

- Dr. Susanne Kammerer

Quelle: Merz Expertentreffen „Vom Profi zum Virtuosen", Frankfurt, Februar 2013 (Veranstalter: Merz)

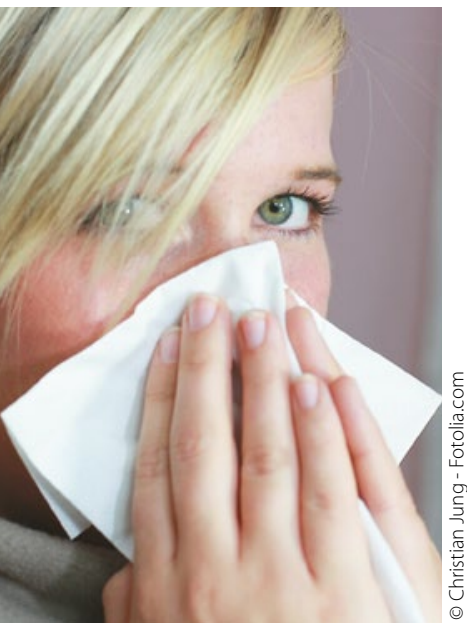

Hypertone Kochsalzlösungen helfen, die Beschwerden bei Rhinosinusitis zu lindern.

\section{Chronische Rhinosinusitis}

\section{Osmotischer Effekt einer Kochsalzlösung unterstützt die Therapie}

- Die spezifische Therapie bei Patienten mit einer chronischen Rhinosinusitis lässt sich durch natürliche Arzneien unterstützen: So kann nach der aktuellen Leitlinie der Deutschen Gesellschaft für Hals-Nasen-OhrenHeilkunde Kopfund Hals-Chirurgie die lokale Anwendung von hypertonen Salzlösungen eine Linderung der Beschwerden bewirken.
Durch den osmotischen Effekt schwellen die Nasenschleimhäute ab, das Nasensekret verflüssigt sich. Folglich werde die mukoziliäre Clearance verbessert, teilt das Unternehmen Bionorica SE mit. So habe in einer kontrollierten, prospektiven Studie mit 211 Patienten die zweimal tägliche Anwendung einer hypertonen Salzlösung zu einer Reduktion der Beschwerden geführt. Und in einem Cochrane-Review sei der Nutzen einer topischen Salzlösung als unterstützende Maßnahme bei chronischer Rhinosinusitis bestätigt worden.

Rinupret ${ }^{\oplus}$-Pflege-Nasenspray enthält außer natürlichem Meersalz in hypertoner Lösung (2,7\%ig) Eukalyptusöl, das belebend wirkt, sowie pflegende Aloe vera. Der
Nasenschleimhaut werde durch die Salzkonzentration im Spray überschüssiges Wasser entzogen, die Schleimhäute schwellen ab. Die frei werdende Flüssigkeit löst zähes Sekret und Verkrustungen. Der Effekt ist lokal und unterstützt die Selbstreinigungs- und Abwehrfunktion der Nasenschleimhaut auch bei längerfristiger Anwendung. Das gut verträgliche Präparat kann bei Erwachsenen und Kindern ab zwei Jahren angewendet werden. Es ist zur begleitenden Behandlung bei Entzündungen der Nasenschleimhaut mit verstopfter Nase oder Schnupfen geeignet.

- Red.

Quelle: nach Informationen des Unternehmens Bionorica SE 